\title{
REVIEW
}

\section{Methotrexate and Rheumatoid Arthritis: Current Evidence Regarding Subcutaneous Versus Oral Routes of Administration}

\author{
Gerolamo Bianchi · Roberto Caporali · Monica Todoerti ·
}

Paolo Mattana

To view enhanced content go to www.advancesintherapy.com

Received: December 22, 2015 / Published online: February 4, 2016

(C) The Author(s) 2016. This article is published with open access at Springerlink.com

\begin{abstract}
Methotrexate (MTX) is still considered the drug of choice in rheumatoid arthritis (RA) management. Comparing subcutaneous (MTX SC) and oral (MTX OR) routes of administration is important to optimize the everyday therapeutic strategy in the real-life setting. This review summarizes scientific evidence currently available on this topic. As shown by pharmacokinetic studies, at the same dose level, bioavailability of MTX SC is significantly higher and less variable than that of MTX OR. This difference is even more pronounced for medium-to-high dosages (i.e., $>15 \mathrm{mg} /$ week). With regard to clinical response (Disease Activity Score-28, American

Electronic supplementary material The online version of this article (doi:10.1007/s12325-016-0295-8) contains supplementary material, which is available to authorized users.
\end{abstract}

G. Bianchi $(\bowtie)$

Division of Rheumatology, ASL3 Genovese, Genoa, Italy

e-mail: gerolamo_bianchi@tin.it;

gerolamo.bianchi@asl3.liguria.it

R. Caporali · M. Todoerti

Division of Rheumatology, IRCCS San Matteo,

University of Pavia, Pavia, Italy

P. Mattana

Division of Medical Service, Alfa Wassermann, Bologna, Italy
College of Rheumatology Criteria), randomized, double-blind studies and retrospective or longitudinal analyses in real-life settings showed that MTX SC is more effective than MTX OR. This is true both in MTX-naive patients with early RA, and in patients who switch from MTX OR to MTX SC due to previous treatment failure, lack of efficacy and/or adverse events. Finally, MTX SC has a better tolerability profile than MTX OR, with fewer gastroenterological side effects. Delaying the use of more expensive biological therapies by switching from MTX OR to MTX SC in non-responders might provide cost savings, with relevant implications in the management of patients with RA.

Funding: Alfa Wassermann.

Keywords: Methotrexate; Oral route; Rheumatoid arthritis; Rheumatology; Subcutaneous route

\section{INTRODUCTION}

Due to its relatively low cost and favorable efficacy/safety profile, methotrexate (MTX) is currently considered the drug of choice for 
treating rheumatoid arthritis (RA), both as first-line monotherapy in treatment-naive patients [1-4], and as an anchor drug, in MTX-insufficient responders, in combination with other conventional (csDMARDs) or biological disease-modifying antirheumatic drugs (bDMARDs) [3-5].

MTX optimization is therefore fundamental in the effective management of patients with RA. In this respect there are several aspects of utmost importance, such as the starting and target doses of MTX, the best treatment strategy [6-8], and the optimal route of administration $[9,10]$. In fact, the choice of MTX route of administration might significantly affect drug bioavailability, thus influencing both its efficacy and tolerability. The aim of this article is to review the available evidence on differences in the pharmacological characteristics and corresponding clinical effects of oral (MTX OR) and subcutaneous MTX (MTX SC) in patients with RA. This review is based on previously conducted studies and does not involve any new studies of human or animal subjects performed by any of the authors.

\section{SEARCH METHODS}

The PubMed and Medline databases were searched up to November 1, 2015 to identify publications on the use of oral and subcutaneous MTX usage in RA. A combination of relevant keywords including methotrexate, subcutaneous route, oral route, rheumatoid arthritis, treatment, pharmacokinetic, efficacy and toxicity were used (Table 1). Publications were hand searched and selected for inclusion in the review based on the authors experience in the field (Table 2).

In particular, the references were first selected from title and abstract, then included or discarded after reading the full text. The main inclusion criteria were studies comparing MTX SC versus MTX OR in patients with RA.

\section{BIOAVAILABILITY}

The existence of different routes of MTX administration (oral and parenteral) has led to the conduction of pharmacokinetic studies designed to compare and highlight any significant differences in the drug's therapeutic impact. Several studies have demonstrated higher bioavailability at various dosages with MTX SC than with MTX OR (Table 3). Differences appear to be especially marked at MTX doses $>15 \mathrm{mg} /$ week.

In 1993, for the first time, Jundt et al. [11] compared the pharmacokinetics of MTX at various dosages in OR, intramuscular (IM) and SC formulations in 12 patients with RA. The authors showed that mean bioavailability was significantly lower with MTX OR than with MTX SC (0.85 vs. $0.97 ; P=0.002$; Table 3$)$, and that there was no significant difference between the two parenteral routes of administration (IM and SC). The authors attributed this difference to intra- and inter-subject variability in the oral absorption of the drug across the gastrointestinal tract. These data were later confirmed in a study comparing the pharmacokinetics of MTX OR and MTX SC at doses $\geq 25 \mathrm{mg}$ /week (range $25-40 \mathrm{mg} /$ week) in 15 patients with RA [12]. MTX OR bioavailability was highly variable and significantly lower (by about one-third; $P=0.001$ ) than that of MTX SC (Table 3).

A more recent randomized, open-label, cross-over trial evaluated the pharmacokinetics of MTX OR and MTX SC over a wide range of doses, corresponding to those commonly used in clinical practice $(10,15,20$, and $25 \mathrm{mg}$ / 
Table 1 Results of literature search

\begin{tabular}{|c|c|c|c|}
\hline Modality of search & $\begin{array}{l}\text { No. references } \\
\text { resulting from } \\
\text { the search }\end{array}$ & $\begin{array}{l}\text { No. references } \\
\text { included in } \\
\text { the study }\end{array}$ & References \\
\hline \multicolumn{4}{|l|}{ PubMed and Medline query } \\
\hline $\begin{array}{l}\text { Methotrexate and subcutaneous } \\
\text { (or parenteral) and oral and } \\
\text { rheumatoid arthritis }\end{array}$ & 108 & 8 & {$[11-17,21,23]$} \\
\hline $\begin{array}{l}\text { Methotrexate and subcutaneous } \\
\text { (or parenteral) and oral and } \\
\text { pharmacokinetic }\end{array}$ & 19 & 2 & {$[12,13]$} \\
\hline $\begin{array}{l}\text { Methotrexate and subcutaneous } \\
\text { (or parenteral) and oral and } \\
\text { efficacy }\end{array}$ & 35 & 4 & {$[12,14,17,21]$} \\
\hline $\begin{array}{l}\text { Methotrexate and subcutaneous } \\
\text { (or parenteral) and oral and } \\
\text { toxicity }\end{array}$ & 55 & 1 & {$[21]$} \\
\hline \multicolumn{4}{|l|}{ Other } \\
\hline $\begin{array}{l}\text { Studies already known by authors } \\
\text { and/or cited in other articles }\end{array}$ & - & 2 & {$[18,19]$} \\
\hline $\begin{array}{l}\text { Reviews, guidelines, other articles } \\
\text { already known by authors and/ } \\
\text { or cited in other articles }\end{array}$ & - & 12 & {$[1-10,20,22]$} \\
\hline
\end{tabular}

Table 2 Characteristics of the literature included in the review

\begin{tabular}{lll}
\hline Kind of study & No. & References \\
\hline Systematic review/guideline & 5 & {$[3,4,7,20,22]$} \\
Systematic review & 5 & {$[2,5,6,9,10]$} \\
Randomized, controlled, double blind & 1 & {$[14]$} \\
Randomized, controlled, open label & 5 & {$[8,11-13,15]$} \\
Prospective cohort study & 1 & {$[21]$} \\
Retrospective & 4 & {$[16-19]$} \\
Cost-minimization analysis & 1 & {$[23]$} \\
Expert opinion/editorial & 1 & {$[1]$}
\end{tabular}

week), in 47 patients with RA [13]. In accordance with the study results, MTX SC bioavailability was higher than that of MTX OR across all tested doses with substantial differences, from $20 \%$ to $40 \%$, between the two routes of administration (+21\%, $+14 \%$, $+31 \%$, and $+41 \%$ for MTX 10, 15, 20, and $25 \mathrm{mg} /$ week, respectively; Table 3). In 
Table 3 Pharmacokinetic differences between MTX SC and MTX OR

\begin{tabular}{lll}
\hline MTX dose range tested & Mean bioavailability & References \\
\hline $7.5-17.5 \mathrm{mg} /$ week & MTX SC: $0.97(95 \%$ CI 0.83-1.12) & Jundt et al. [11] \\
& MTX OR: 0.85 (95\% CI 0.77-0.93) & Hoekstra et al. [12] \\
$25-40 \mathrm{mg} /$ week & MTX SC: 1.0 & \\
& MTX OR: 0.64 (range 0.21-0.96) & \\
$10-25 \mathrm{mg} /$ week & Between group differences (MTX SC vs. MTX OR) & Schiff et al. [13] \\
& MTX $10 \mathrm{mg}:+21 \%$ & \\
& MTX $15 \mathrm{mg}:+14 \%$ & \\
& MTX 20 mg: $+31 \%$ & \\
& MTX 25 mg: $+41 \%$ & \\
\hline
\end{tabular}

$C I$ confidence intervals, $M T X$ methotrexate, $O R$ oral, $S C$ subcutaneous

a $P=0.002$ vs. MTX OR

b $P=0.001$ vs. MTX SC

particular, a plateau effect was demonstrated for MTX OR at doses above $15 \mathrm{mg} /$ week, whilst MTX SC showed a linear, dose-proportional increase, with no ceiling effect up to the highest dose of $25 \mathrm{mg} /$ week.

\section{EFFICACY AND TOLERABILITY}

Growing evidence supports greater efficacy (Tables 4, 5) and improved tolerability with MTX SC compared with MTX OR in RA management.

\section{Clinical Trial Data}

A multicenter, randomized, double-blind trial assessed MTX efficacy and tolerability in 381 patients with active early RA (Disease Activity Score-28 [DAS28] of $>4$ ), who were MTX-naive [14]. At baseline, patients were randomized to receive MTX OR or MTX SC at $15 \mathrm{mg} /$ week, then if clinical response [defined as a $20 \%$ improvement in American College of Rheumatology Criteria (ACR20)] was not achieved at week 16, patients originally

Table 4 Differences in clinical response between MTX SC and MTX OR

\begin{tabular}{llllll}
\hline Outcome & $\begin{array}{l}\text { Duration of therapy, } \\
\text { months }^{\mathbf{a}}\end{array}$ & MTX SC & MTX OR & P value & References \\
\hline ACR20, \% & 6 & $78 \%$ & $70 \%$ & $<0.05$ & Braun et al. [14] \\
ACR70, \% & 6 & $41 \%$ & $33 \%$ & $<0.05$ & \\
DAS28, mean (SD) & 3 & $3.49(1.50)$ & $3.92(1.48)$ & 0.002 & Hazlewood et al. [21] \\
DAS28, mean (SD) & 6 & $3.12(1.46)$ & $3.50(1.51)$ & 0.011 & \\
DAS28, mean (SD) & 9 & $2.79(1.37)$ & $3.23(1.53)$ & 0.005 & \\
Change of therapy, \% & 12 & $49 \%$ & $77 \%$ & $<0.001$ & Hazlewood et al. [21] \\
\hline
\end{tabular}

$A C R$ American College of Rheumatology, DAS Disease Activity Score, $M T X$ methotrexate, $O R$ oral, $S C$ subcutaneous, $S D$ standard deviation 
Table 5 Clinical response in patients switched from MTX OR to MTX SC because of treatment failure (inefficacy or toxicity)

\begin{tabular}{llll}
\hline Outcome & $\begin{array}{l}\text { Duration of } \\
\text { therapy, months }\end{array}$ & MTX SC & References \\
\hline ACR20, \% & 2 & 30 & Braun et al. [14] \\
Mean reduction in DAS28 score & 1 & 0.3 & Bakker et al. [15] \\
& 4 & 0.5 & Mainmann et al. [16] \\
DAS28 reduction of $\geq 1.2$ points, $\%$ & 6 & 74 & Bakker et al. [15] \\
DAS28 score $<3.2, \%$ & 6 & 29 & Scott et al. [17] \\
Improvement of DAS28, \% & 4 & 63 & \\
Continuation rate, \% & 12 & 83 & Scott et al. [17] \\
& 24 & 75 & \\
\hline
\end{tabular}

$A C R$ American College of Rheumatology, DAS Disease Activity Score, MTX methotrexate; OR oral, SC subcutaneous

assigned to MTX OR were switched to MTX SC at the same dose (15 mg/week), while those randomized to MTX SC "stepped-up" from 15 to $20 \mathrm{mg} /$ week. After 6 months of treatment, clinical response was significantly higher in the MTX SC group than in the MTX OR group (ACR20, 78\% vs. 70\%; ACR70, 41\% vs. 33\%; Table 4). Inter-group differences were already significant at 16 weeks of observation. After 16 weeks of treatment, overall 52 patients (14\%) were non-responders. ACR20 was subsequently achieved in 23\% MTX SC recipients after a dose increase from 15 to $20 \mathrm{mg} /$ week, and in 30\% of MTX OR recipients after switching to MTX SC (Table 5). There was no significant difference in side effects between the two treatment groups.

The CAMERA study (Computer Assisted Management in Early RA) examined the efficacy and tolerability of a conventional MTX treatment approach compared with an intensive treatment strategy, which involved rapid stepwise increases in MTX OR dose by $5 \mathrm{mg} /$ week every month up to a maximum dose of $30 \mathrm{mg} /$ week, with a switch to the same dose of MTX SC in case of poor response or intolerable adverse events, plus a further step of addition of cyclosporine to MTX SC $15 \mathrm{mg} /$ week [8]. A post hoc analysis of this study, focused on the switch from MTX OR to MTX SC, specifically in the subset of patients originally assigned to the intensive strategy, has been published [15]. Data from 57 of the 151 patients originally randomized to the intensive therapy arm were analyzed: 21 patients switched from MTX OR to MTX SC due to adverse events and 36 patients switched due to inadequate clinical efficacy. Regardless of the reason for switching, a statistically significant reduction in the mean value of DAS28 was seen 1 month after the switch, and for up to 4 months of evaluation $(P<0.05$; Table 5$)$.

The benefits of switching MTX route of administration, from oral to parenteral, have 
also been confirmed in patients with long-standing disease. In a retrospective analysis, 78 patients receiving MTX SC treatment, all of whom had previously failed MTX OR therapy because of lack of efficacy or side effects, were compared with 78 control patients, responders to MTX OR monotherapy, matched for disease duration (mean 5 years) and baseline DAS scores [16]. Six months after the switch, patients receiving MTX SC experienced a significant improvement from baseline in DAS28 scores, and showed final levels of disease activity comparable with responders to MTX OR (Table 5).

More recently, the MENTOR study (Methotrexate Evaluation of Norwich Treatment Outcomes in RA) evaluated the short-, medium- and long-term effects (up to 5 years of follow-up) of a therapeutic switch from MTX OR to MTX SC, due to intolerance (43.9\% of patients) or inefficacy $(50.5 \%$ of patients), in a cohort of 196 patients with long-standing RA [17]. The switch to MTX SC provided either a good long-term survival on therapy (retention rates of $83 \%$ at 1 year, $75 \%$ at 2 years, and $47 \%$ at 5 years), and a minimal need for further treatment with bDMARDs $(<10 \%$; Table 5). Based on these results, the authors suggested that the parenteral route of administration should always be considered before determining a patient's overall failure on or intolerance to MTX. In other words, failure of MTX therapy should only be declared in case of lack of efficacy of or intolerance to MTX SC.

In addition, MTX SC seems to ensure better tolerability and safety profiles, mainly with less gastrointestinal discomfort for patients, compared with MTX OR. Rutkowska-Sak et al. [18] conducted an observational study based on a patient-based questionnaire focusing on gastrointestinal toxicity with MTX. A total of
70 patients with RA were initially treated with MTX OR (7.5 or $15 \mathrm{mg} /$ week), but switched to MTX SC at the same dose because of side effects. At both dosage levels, the switch from MTX OR to MTX SC resulted in a marked improvement in the subjective tolerability profile of the drug with a significant reduction in the intensity of nausea and abdominal pain and the disappearance of vomiting and diarrhea.

\section{Real-Life Data}

Recently, the efficacy and safety data of MTX SC have also been confirmed in several real-life studies, far away from the controlled and selected settings of clinical trials. A retrospective analysis was conducted on a cohort of 70 patients with RA in the St. Gallen hospital in Switzerland who were MTX- and csDMARD-naive, with the aim of evaluating the efficacy, safety and tolerability of MTX SC in real life [19]. Patients were initially treated with MTX SC at various dosages ranging from 10 to $25 \mathrm{mg} /$ week, according to treat-to-target and tight control principles recommended in current EULAR guidelines [20]. During the mean observation period of 1.8 years, 37 patients (53\%) continued MTX SC monotherapy with clinical benefit, while 33 patients $(47 \%)$ required addition of a bDMARD due to incomplete disease control [19]. On average, the use of the bDMARDs was necessary in almost half of the patients after approximately 1 year of treatment with MTX SC (mean 387 days). Overall, most patients achieved disease control [80\% of patients had low disease activity (LDA) and 73\% achieved clinical remission according to DAS28 score]. Furthermore, this result was achieved more rapidly in the group treated with MTX SC alone than in the group that required combination therapy with bDMARDs: among 
patients treated with MTX SC monotherapy, LDA was achieved within a mean of 111 days in $81.1 \%$ of patients, compared with a mean of 198 days in $78.8 \%$ of patients treated with MTX + bDMARDs. Similarly, DAS28 remission was achieved by $75.7 \%$ of patients after a mean of 145 days of MTX monotherapy, compared with $69.7 \%$ of patients after a mean of 297 days of MTX + bDMARDs. During the study, the discontinuation rate and the incidence of adverse events were similar for both groups, regardless of the addition of bDMARDs.

More recently, Hazlewood and co-authors [21] compared the overall effectiveness of MTX OR with MTX SC as initial therapy in 666 patients with early RA (symptoms for less than 1 year). Patients were originally enrolled in a multicenter, observational study in which the choice of MTX route of administration was at the discretion of the treating rheumatologist. After 1 year of treatment a significantly higher proportion of patients initially treated with MTX OR $(n=417)$ had treatment failure compared with those who received MTX SC ( $n=249 ; 77 \%$ vs. $49 \%$, respectively; Table 4$)$, mostly due to lack of efficacy rather than toxicity or intolerance to therapy. More specifically, patients originally treated with MTX OR had to increase the MTX dose, add or switch to bDMARDs, and change the route of administration much more frequently than patients treated with MTX SC. After adjustment for potential confounders related to the non-randomized assignment of drug formulation, MTX SC was closely linked to a lower probability of global treatment failure [hazard ratio $0.55 ; 95 \%$ confidence interval (CI) 0.39-0.79], justified primarily by a lower probability of ineffectiveness of the drug. Patients treated with MTX SC showed a more significant reduction in mean DAS28 values at 3,6 , and 9 months after the beginning of treatment compared with patients treated with MTX OR (Table 4), with a significantly higher probability of obtaining DAS28 clinical remission (odds ratio 1.15; 95\% CI 1.05-1.25; $P=0.002)$.

\section{DISCUSSION}

Bioavailability data discussed herein are reflected in the latest Italian recommendations and guidelines on the management of RA [7, 22]. While guidelines permit a broad therapeutic range $(7.5-25.0 \mathrm{mg} /$ week $)$, the optimal treatment strategy requires initial doses higher than those recommended in the past (12.5-15.0 mg/week). In addition, a specific focus on MTX administered parenterally has been added, with guidelines recommending MTX SC both as first-line therapy and in patients refractory to MTX OR, to ensure higher bioavailability and thus achieve greater clinical efficacy.

In support of this, clinical trial data demonstrate that: (1) as initial therapy, MTX $\mathrm{SC}$ is associated with greater clinical efficacy than MTX OR at the same dosage level of $15 \mathrm{mg} /$ week in MTX-naive patients with early RA; (2) in cases of failure with MTX OR, switching to MTX SC at the same dosage is associated with attainment of a clinical response in up to one-third more patients, with no apparent increase in adverse effects/toxicity; (3) in cases of no response to MTX SC, dose step-up was efficacious and safe [14]. The greater efficacy and flexibility of MTX SC compared with MTX OR is mostly due to its higher bioavailability at all possible dosages, but especially at medium-high doses [11-13]. Real-life data also support the use of MTX SC in routine care. MTX SC was shown to be an effective and well-tolerated primary alternative 
to MTX OR in everyday clinical practice for patients with early RA $[19,21]$.

Switching to MTX SC might also limit and delay the eventual need for further therapies, with the possibility of an increased risk in terms of adverse effects and costs. To this end, a recent study examined the theoretical economic impact of MTX SC compared with biological drugs over a 12-month period in a hypothetical population of RA patients who had failed to respond to MTX OR [23]. The economic model was calculated using published epidemiological and clinical data, and referred to the National Institute for Health and Clinical Excellence guidelines regarding the labeled use of bDMARDs. On this hypothetical background, in the UK, the routine use of MTX SC after failure of MTX OR could potentially lead to an estimated savings of $£ 7197$ per patient in the first year of therapy and $£ 9.3$ million a year nationally in new patients, due to the reduced use of biological drugs [23].

\section{CONCLUSIONS}

In conclusion, in accordance with the latest scientific evidence on the topic of MTX and RA, we might consider the following observations:

1. From a pharmacokinetic standpoint, at the same dosage, MTX SC has a significantly higher bioavailability with respect to the oral route of administration. This difference is even more pronounced for medium-to-high dosages (i.e., $>15 \mathrm{mg} /$ week).

2. MTX SC is a more flexible therapeutic option than MTX OR, with a greater potential in terms of dosage range. MTX OR shows a plateau effect for dosages higher than $15 \mathrm{mg} /$ week, whilst MTX SC exhibits a linear dose-dependent increase in bioavailability up to high dosages $(25 \mathrm{mg}$ / week).
3. From a therapeutic standpoint, both clinical trials and real-life studies demonstrate that MTX SC is superior to MTX OR in terms of clinical efficacy (DAS28 and ACR response), either as first-line therapy in MTX-naive patients, or in MTX OR-experienced patients as switch therapy. Clinical benefit has been demonstrated in both early and long-standing disease.

4. MTX SC also shows a better tolerability profile with respect to gastrointestinal toxicity. Other side effects are similar between parenteral and oral routes.

5. Overall, MTX SC is characterized by higher bioavailability, greater clinical efficacy, and a better tolerability profile than MTX OR.

Thus, current evidence suggests that the choice of MTX route of administration is a fundamental parameter for optimizing RA treatment. The SC route of administration seems to be the best treatment option from the outset, in terms of risk:benefit ratio compared with the oral route. Finally, although to date there are no real evidence of cost-effectiveness of MTX SC versus MTC OR, it is possible to assume that delaying the use of more aggressive and more expensive therapies, such as bDMARDs, by switching from MTX OR to MTX SC in non-responders might provide cost savings while reducing risks, with relevant implications in the everyday clinical care of patients with RA. In this regard, a specifically designed study to assess this outcome would be desirable.

\section{ACKNOWLEDGMENTS}

All named authors meet the International Committee of Medical Journal Editors (ICMJE) criteria for authorship for this manuscript, take responsibility for the integrity of the work as a whole, and have given final approval to the 
version to be published. Native English and technical editing of this manuscript was performed by Andrea Bothwell on behalf of Springer Healthcare Communications, and was funded by Alfa Wassermann, Italy. The article processing charges for this publication were funded by Alfa Wassermann.

Disclosures. Gerolamo Bianchi, Roberto Caporali, and Monica Todoerti declare that they have no conflicts of interest. Paolo Mattana is an employee of Alfa Wassermann.

Compliance with Ethics Guidelines. This article is based on previously conducted studies, and does not involve any new studies of human or animal subjects performed by any of the authors.

Open Access. This article is distributed under the terms of the Creative Commons Attribution-NonCommercial 4.0 International License (http://creativecommons.org/licenses/ by-nc/4.0/), which permits any noncommercial use, distribution, and reproduction in any medium, provided you give appropriate credit to the original author(s) and the source, provide a link to the Creative Commons license, and indicate if changes were made.

\section{REFERENCES}

1. Bijlsma JW, Jacobs JW. Methotrexate: still the anchor drug in RA treatment. Jt Bone Spine. 2009;76(5):452-4.

2. Lopez-Olivo MA, Siddhanamatha HR, Shea B, Tugwell P, Wells GA, Suarez-Almazor ME. Methotrexate for treating rheumatoid arthritis. Cochrane Database Syst Rev. 2014;6:CD000957.

3. Singh JA, Furst DE, Bharat A, et al. 2012 update of the 2008 American College of Rheumatology recommendations for the use of disease-modifying antirheumatic drugs and biologic agents in the treatment of rheumatoid arthritis. Arthritis Care Res (Hoboken). 2012;64(5):625-39.

4. Smolen JS, Landewe R, Breedveld FC, et al. EULAR recommendations for the management of rheumatoid arthritis with synthetic and biological disease-modifying antirheumatic drugs. Ann Rheum Dis. 2010;69(6):964-75.

5. Emery P, Sebba A, Huizinga TW. Biologic and oral disease-modifying antirheumatic drug monotherapy in rheumatoid arthritis. Ann Rheum Dis. 2013;72(12):1897-904.

6. Mouterde G, Baillet A, Gaujoux-Viala C, et al. Optimizing methotrexate therapy in rheumatoid arthritis: a systematic literature review. Jt Bone Spine. 2011;78(6):587-92.

7. Todoerti M, Maglione W, Bernero E, et al. Systematic review of 2008-2012 literature and update of recommendations for the use of methotrexate in rheumatic diseases, with a focus on rheumatoid arthritis. Reumatismo. 2013;65(5):207-18.

8. Verstappen SM, Jacobs JW, van der Veen MJ, et al. Intensive treatment with methotrexate in early rheumatoid arthritis: aiming for remission. Computer Assisted Management in Early Rheumatoid Arthritis (CAMERA, an open-label strategy trial). Ann Rheum Dis. 2007;66(11):1443-9.

9. Cipriani P, Ruscitti P, Carubbi F, Liakouli V, Giacomelli R. Methotrexate in rheumatoid arthritis: optimizing therapy among different formulations. Current and emerging paradigms. Clin Ther. 2014;36(3):427-35.

10. Visser K, van der Heijde D. Optimal dosage and route of administration of methotrexate in rheumatoid arthritis: a systematic review of the literature. Ann Rheum Dis. 2009;68(7):1094-9.

11. Jundt JW, Browne BA, Fiocco GP, Steele AD, Mock D. A comparison of low dose methotrexate bioavailability: oral solution, oral tablet, subcutaneous and intramuscular dosing. J Rheumatol. 1993;20(11):1845-9.

12. Hoekstra M, Haagsma C, Neef C, Proost J, Knuif A, van de Laar M. Bioavailability of higher dose methotrexate comparing oral and subcutaneous administration in patients with rheumatoid arthritis. J Rheumatol. 2004;31(4):645-8.

13. Schiff MH, Jaffe JS, Freundlich B. Head-to-head, randomised, crossover study of oral versus subcutaneous methotrexate in patients with rheumatoid arthritis: drug-exposure limitations of oral methotrexate at doses $\geq 15 \mathrm{mg}$ may be 
overcome with subcutaneous administration. Ann Rheum Dis. 2014;73(8):1549-51.

14. Braun J, Kastner P, Flaxenberg P, et al. Comparison of the clinical efficacy and safety of subcutaneous versus oral administration of methotrexate in patients with active rheumatoid arthritis: results of a six-month, multicenter, randomized, double-blind, controlled, phase IV trial. Arthritis Rheum. 2008;58(1):73-81.

15. Bakker MF, Jacobs JW, Welsing PM, et al. Are switches from oral to subcutaneous methotrexate or addition of ciclosporin to methotrexate useful steps in a tight control treatment strategy for rheumatoid arthritis? A post hoc analysis of the CAMERA study. Ann Rheum Dis. 2010;69(10):1849-52.

16. Mainman H, McClaren E, Heycock C, Saravanan V, Hamilton J, Kelly C. When should we use parenteral methotrexate? Clin Rheumatol. 2010;29(10):1093-8.

17. Scott DG, Claydon P, Ellis C. Retrospective evaluation of continuation rates following a switch to subcutaneous methotrexate in rheumatoid arthritis patients failing to respond to or tolerate oral methotrexate: the MENTOR study. Scand J Rheumatol. 2014;43(6):470-6.

18. Rutkowska-Sak L, Rell-Bakalarska M, Lisowska B. Oral vs. subcutaneous low-dose methotrexate treatment in reducing gastrointestinal side effects. Reumatologia. 2009;47(7):207-11.
19. Muller RB, von Kempis J, Haile SR, Schiff MH. Effectiveness, tolerability, and safety of subcutaneous methotrexate in early rheumatoid arthritis: a retrospective analysis of real-world data from the St. Gallen cohort. Semin Arthritis Rheum. 2015;45(1):28-34.

20. Smolen JS, Landewe R, Breedveld FC, et al. EULAR recommendations for the management of rheumatoid arthritis with synthetic and biological disease-modifying antirheumatic drugs: 2013 update. Ann Rheum Dis. 2014;73(3):492-509.

21. Hazlewood GS, Thorne JC, Pope JE, et al. The comparative effectiveness of oral versus subcutaneous methotrexate for the treatment of early rheumatoid arthritis. Ann Rheum Dis. 2015. pii: annrheumdis-2014-206504. doi:10.1136/ annrheumdis-2014-206504 [Epub ahead of print].

22. De Leonardis F, Alivernini S, Bonacci E, et al. Italian consensus on the recommendations about the use of methotrexate for the treatment of rheumatic diseases with a focus on rheumatoid arthritis: results from the "3E initiative". Reumatismo. 2010;62(1):34-45.

23. Fitzpatrick R, Scott DG, Keary I. Cost-minimisation analysis of subcutaneous methotrexate versus biologic therapy for the treatment of patients with rheumatoid arthritis who have had an insufficient response or intolerance to oral methotrexate. Clin Rheumatol. 2013;32(11):1605-12. 